\title{
Acetone utilization by sulfate-reducing bacteria: draft genome sequence of Desulfococcus biacutus and a proteomic survey of acetone-inducible proteins
}

\author{
Olga B Gutiérrez Acosta, David Schleheck and Bernhard Schink
}

\begin{abstract}
Background: The sulfate-reducing bacterium Desulfococcus biacutus is able to utilize acetone for growth by an inducible degradation pathway that involves a novel activation reaction for acetone with CO as a co-substrate. The mechanism, enzyme(s) and gene(s) involved in this acetone activation reaction are of great interest because they represent a novel and yet undefined type of activation reaction under strictly anoxic conditions.

Results: In this study, a draft genome sequence of $D$. biacutus was established. Sequencing, assembly and annotation resulted in 159 contigs with 5,242,029 base pairs and 4773 predicted genes; 4708 were predicted protein-encoding genes, and 3520 of these had a functional prediction. Proteins and genes were identified that are specifically induced during growth with acetone. A thiamine diphosphate-requiring enzyme appeared to be highly induced during growth with acetone and is probably involved in the activation reaction. Moreover, a coenzyme $B_{12}$ - dependent enzyme and proteins that are involved in redox reactions were also induced during growth with acetone.

Conclusions: We present for the first time the genome of a sulfate reducer that is able to grow with acetone. The genome information of this organism represents an important tool for the elucidation of a novel reaction mechanism that is employed by a sulfate reducer in acetone activation.
\end{abstract}

Keywords: Desulfococcus biacutus, Acetone activation, Genome sequencing, Carbonylation, Thiamine diphosphate

\section{Background}

Aerobic and nitrate-reducing bacteria can utilize acetone for growth through activation of the acetone molecule by an endergonic carboxylation reaction to form acetoacetate as a first reaction product [1-3]. For example, the respective enzyme in Xanthobacter strain Py2, acetone carboxylase (EC 6.4.1.6) [1,4], activates acetone with $\mathrm{CO}_{2}$ to form acetoacetate at the expense of ATP. ATP is hydrolyzed to AMP plus pyrophosphate which is later hydrolyzed to two phosphates, hence, the reaction consumes two ATP equivalents if the regeneration of ATP from AMP is taken into account. The acetone carboxylase of the nitrate reducer Aromatoleum aromaticum [2] activates acetone with bicarbonate at the expense of two

\footnotetext{
*Correspondence: bernhard.schink@uni-konstanz.de

Department of Biology and Konstanz Research School Chemical Biology, University of Konstanz, D-78457 Konstanz, Germany
}

ATP that are hydrolyzed to two AMP plus four phosphate, hence, effectively at the expense of even four ATP equivalents. Aerobic and nitrate-reducing bacteria can gain sufficient energy from the oxidation of acetoacetate to sustain this energy input required for the initial carboxylation of acetone, in addition to the energy input required to sustain their growth.

Acetone activation by the strictly anaerobic, dissimilatory sulfate-reducing bacteria (SRB) has not been studied in detail yet, and it is unknown how these bacteria perform the endergonic activation reaction. The overall energy derived from acetoacetate oxidation with sulfate as electron acceptor (that is, about two to three mol ATP per mol acetoacetate), appears to be hardly sufficient to support growth and activation of acetone via any of the carboxylase reactions described above. Up to date, two acetone-utilizing SRB are known, the deltaproteobacteria 
Desulfobacterium cetonicum [5] and Desulfococcus biacutus strain KMRActS [6]; the latter bacterium has been isolated from an anaerobic sludge digestor of a wastewater treatment plant.

Recently, we proposed that $D$. biacutus activates acetone in a carbonylation reaction to form acetoacetaldehyde [7]. The reaction was followed in cell-free extracts of acetone-grown $D$. biacutus and requires ATP as a cofactor, and $\mathrm{CO}$ rather than $\mathrm{CO}_{2}$ as a co-substrate [7]. The reaction product acetoacetaldehyde was trapped as the dinitrophenyl-hydrazone derivative and identified by mass spectrometry after a reaction of acetone, CO, ATP, and dinitrophenylhydrazine with cell-free extracts [7]. The reaction could not be observed with cell-free extract of butyrate-grown $D$. biacutus, hence, the proposed acetone-activating enzyme appeared to be inducible. However, this novel reaction, and particularly the postulated enzyme(s) and gene(s) involved, remained undefined.

Complete genome sequences of several SRB have become available in the recent years, for example, of the unclassified deltaproteobacterium strain NaphS2 that is able to utilize naphthalene [8], of Desulfobacula toluolica strain Tol2 that grows with aromatic compounds [9], of Desulfobacterium autotrophicum strain HRM2 that is able to utilize fatty acids [10], of Desulfatibacillum alkenivorans AK-01 that utilizes hexadecane [11], and of Desulfotalea psychrophila that uses lactate and alcohols [12], as well as of Desulfotomaculum acetoxidans [13] and of a Desulfarculus baarsii strain [14]. Only one genome of a member of the genus Desulfococcus has been made available thus far, i.e. Desulfococcus oleovorans strain Hxd3 (JGI project id: 4002948) which utilizes $C_{12}-C_{20}$ alkanes for growth [15].

In the present paper, we report the draft-genome sequencing and annotation of the first genome of an acetone-degrading, sulfate-reducing deltaproteobacterium, i.e., of Desulfocccus biacutus strain KMRActS, and hence, of a second member of the genus Desulfococcus. The genome information was used in a differentialproteomics approach to identify genes that are specifically expressed during growth with acetone, in comparison to growth with butyrate. Candidate genes were identified that could be involved in the activation and degradation of acetone in D. biacutus.

\section{Results and discussion}

\section{General features of the Desulfocccus biacutus genome sequence}

Genomic DNA of D. biacutus KMRActS was extracted and submitted to whole-genome shotgun sequencing using a Roche GS FLX + system (see Methods). Sequencing and assembly resulted in a genome of a total size of 5,242,029 bp distributed over 159 contigs. The contigs were annotated via the Integrated Microbial Genomes
(IMG) pipeline (Table 1). In total, 4773 open reading frames (ORFs) were predicted, of which 4708 (98.6\%) were protein-encoding genes, 3520 (73.8\%) genes coding for proteins with a predicted function, and 1128 (23.6\%) were attributed to encode transmembrane proteins; only one set of rRNA genes was found.

In direct comparison to its closest genome-sequenced relative, Desulfococcus oleovorans strain Hxd3, the D. biactus genome appeared to be larger (5.24 Mb vs. 3.94 Mb, respectively; 4773 vs. 3320 ORFs) and, most strikingly, contained a much higher number of assigned transporter genes (606 candidate genes) compared to D. oleovorans (299 candidates); for example, more candidates of the ABC-superfamily (TC:3.A.1) (218 in D. biacutus vs. 77 in D. oleovorans), MFS-superfamily (TC:2.A.1) (17 vs. 8), and TRAP-family (TC:2.A.56) (21 vs. 12). Furthermore, a higher abundance of genes for signal transduction and regulation was found, e.g., for signal transduction histidine kinases (COG4585 and 0642) (43 candidates in D. biacutus vs. 18 in $D$. oleovorans), CheY-like response regulators (COG2197 and 2204) (73 vs. 43), and PAS-containing transcriptional regulators (COG3829) (11 vs. 5). In total, eight CRISPR elements were predicted for $D$. biacutus, and two for $D$. oleovorans. In contrast, the genome of $D$. biacutus harbors less candidate genes for fatty-acid metabolism, e.g., for acyl-CoA dehydrogenases (COG1960) (12 candidates in D. biacutus vs. 25 in D. oleovorans), enoylCoA hydratases (COG1024) (8 vs. 16), and acyl-CoA

Table 1 Statistics of the IMG genome annotation of Desulfococcus biacutus

\begin{tabular}{lrr}
\hline & Number & \% of Total \\
\hline DNA, total number of bases & 5242029 & $100.00 \%$ \\
DNA coding number of bases & 4646037 & $88.63 \%$ \\
DNA G + C number of bases & 3055509 & $58.29 \%$ \\
DNA scaffolds & 159 & $100.00 \%$ \\
CRISPR Count & 8 & \\
Genes total number & 4773 & $100.00 \%$ \\
Protein-encoding genes & 4708 & $98.64 \%$ \\
RNA genes & 65 & $1.36 \%$ \\
rRNA genes & 3 & $0.06 \%$ \\
5S rRNA & 1 & $0.02 \%$ \\
16S rRNA & 1 & $0.02 \%$ \\
23S rRNA & 1 & $0.02 \%$ \\
tRNA genes & 51 & $1.07 \%$ \\
Other RNA genes & 11 & $0.23 \%$ \\
Protein-encoding genes with function prediction & 3520 & $73.75 \%$ \\
Protein coding genes coding transmembrane & 1128 & $23.63 \%$ \\
proteins & & \\
Protein coding genes connected to transporter & 606 & $12.70 \%$ \\
classification & & \\
\hline
\end{tabular}


acetyl-transferase (COG0183) (8 vs. 13), with the exception of acyl-CoA synthetase candidates (COG0318 and 1042) (27 vs.18). The $D$. biacutus genome annotation also indicated a lower abundance of benzoyl-CoA reductase candidates (COG1775) (4 vs. 10 candidates in D. oleovorans). No valid candidate genes for alpha subunits of benzyl-, alkyl-, or naphthylsuccinate synthase (searching with BssA [O87943], AssA1 [B8FEM4] and NsmA [D2XBH8]) were found in either the D. biacutus or D. oleovorans genome, but glycyl-radical enzyme candidate genes were found that most likely represent alpha-subunit genes for pyruvate formate lyases (e.g., DebiaDRAFT_01145). Finally, we found no valid gene candidates for acetone carboxylase in the D. biacutus genome (see below).

\section{Differential proteomics approach}

We aimed at an identification of all proteins that are specifically synthesized in D. biacutus during growth with acetone, in comparison to cells grown with butyrate. Therefore, the annotated draft-genome sequence of $D$. biacutus was used to generate a reference database for peptide mass fingerprinting (PMF). Soluble proteins and membrane proteins were analyzed separately by two-dimensional (2D) and 1D-PAGE, respectively (see Methods), and protein bands or spots of interest were excised for PMF identification. A differential total proteome analysis (Orbitrap-MS analysis) of all proteins in crude extracts (soluble and membrane proteins) was also performed in order to confirm and expand on the identifications made by the gel-based proteomics approach; the results can be found in the Additional files 1: Table S1 and Additional file 2: Table S2 published with this article.

\section{Proteins that were specifically formed during growth with acetone}

Proteins formed specifically during growth with acetone were separated by 2D-PAGE for the soluble protein fraction (Figure 1A,B) and by 1D-PAGE for the membrane fraction (Figure 2). The gels showed seven prominent spots in the cytoplasmic and three in the membrane fraction that were visible only on gels of acetone-grown cells but not on the gels of butyrate-grown cells (as indicated in Figures 1A,B and 2), and were excised and identified (Table 2). Furthermore, fifteen prominent cytoplasmic and five membrane-derived proteins observed on both gels, hence constitutively expressed proteins, were also excised and identified by PMF (Table 3). Five of the genes encoding the seven apparently acetone-inducible, soluble proteins (2D-PAGE spots) were found to be located on short contigs (app. $15 \mathrm{~kb}$ each) termed gene cluster A and B (see Figure 3A,B).

The PMF of prominent spot AS_3 (Figure 1A) corresponded to locus tag (ORF) DebiaDRAFT_04566 (Table 2) in gene cluster A (Figure 3A) (in the following, the locus tag prefixes, DebiaDRAFT_, are omitted). ORF 04566 was also identified by the PMF of an acetone-inducible protein that was observed by 1D-PAGE in the membrane fraction as band AM_2. Notably, the preparation of the membrane fraction involved four washing steps, hence, it is unlikely that band AM_2 resulted from a soluble-protein contamination of the membrane preparation. Moreover, when comparing the intensity of the 2D-PAGE spot (AS_3) and 1D-PAGE band (AM_2) it appears that this enzyme is represented more prominently in the membrane than in the soluble protein fraction. However, for this protein no transmembrane helices or signal peptide sequences were predicted by IMG. Gene 04566 is annotated to encode a thiamine diphosphate (TDP)-requiring enzyme (COG0028; acetolactate synthase/pyruvate dehydrogenase [cytochrome]/glyoxylate carboligase/phosphonopyruvate decarboxylase). The identification of this TDP-enzyme and its induction during growth with acetone was confirmed in a replicate 2D-PAGE, when starting from an independent growth experiment (not shown), and by the total proteome analysis (Orbitrap-MS; see Additional file 1: Table S1).

The detection of this TDP enzyme suggests that TDP might play a key role as a cofactor in the initial acetone activation. TDP is known to participate in carbon-carbon bond-forming or bond-breaking reactions, especially with those substrates that contain a carbonyl group [16-21]. In a separate study employing a fluorescent ATP analogue as probe [22], we could show that ATP is converted to AMP plus pyrophosphate, and that this reaction is stimulated by the presence of TDP. One could speculate that either the central carbon of (activated?) acetone is bound to the ilyl carbon of TDP for the subsequent carbonylation, or that TDP first binds the $\mathrm{CO}$ before the carbonylation of activated acetone.

Two genes that are located on the same contig, that is, in gene cluster $\mathrm{A}$ together with the TDP-enzyme gene 04566 (Figure 3A), were found to encode other proteins that were specifically induced during growth with acetone. One is ORF 04571, identified by the prominent spot AS_17 (Figure 1A) which is annotated to encode a short-chain dehydrogenase/reductase protein (Table 2); these types of dehydrogenases/reductases (COG1028) possess at least two domains, one that binds $\mathrm{NAD}^{+} / \mathrm{NADH}$ or $\mathrm{NADP}^{+} / \mathrm{NADPH}$, and another one that determines the substrate specificity and catalysis. This enzyme might represent an $\mathrm{NAD}^{+}$-dependent dehydrogenase that oxidizes acetoacetaldehyde to acetoacetyl-CoA [7]. The identification and induction of this gene during growth with acetone was also confirmed by the total proteome analysis (see Additional file 1: Table S1). The other acetone-induced gene in cluster A is ORF 04573, identified by the prominent protein spot AS_25 (Figure 1A), which is annotated as the cobalamin $\left(\mathrm{B}_{12}\right)$-binding subunit 


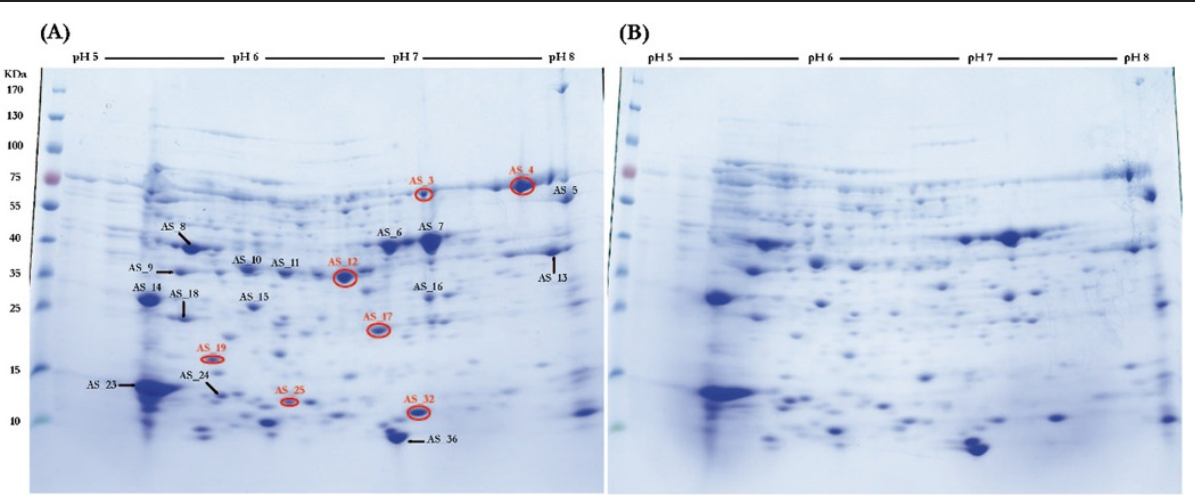

Figure 1 2D-PAGE analysis of soluble proteins produced during growth with (A) acetone and (B) butyrate. The protein spots that were identified by peptide mass fingerprinting (see Tables 2 and 3) are labeled in the acetone gel. Acetone-induced proteins are marked in red. M: molecular mass marker.

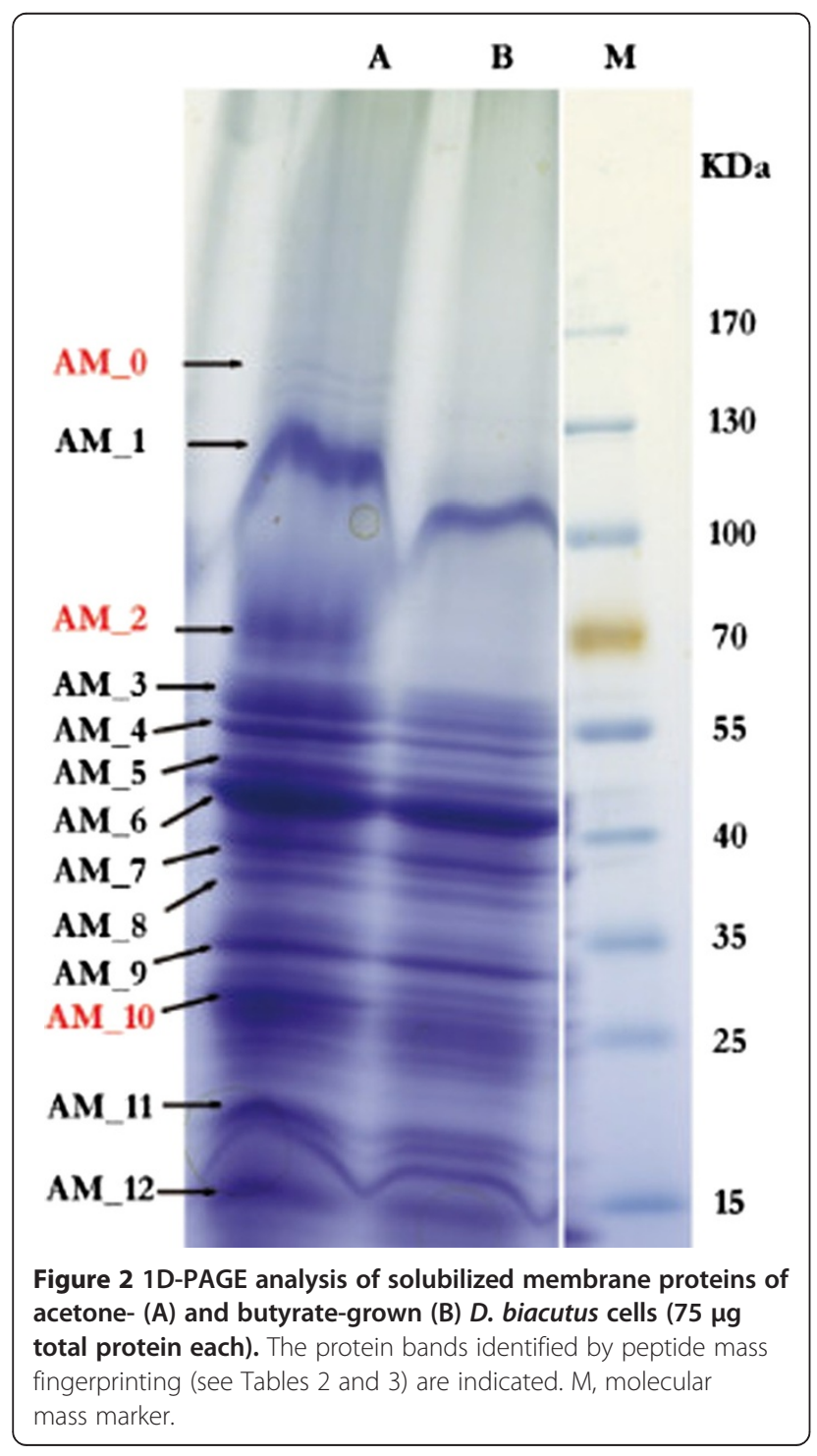

(COG2185) of a methylmalonyl-CoA mutase enzyme complex (Table 2). This gene identification and the inducible expression was also confirmed by the total proteome analysis, in which also the N-terminal methylmalonylCoA mutase subunit (gene 04574) was identified (see Additional file 1: Table S1). This enzyme complex performs various types of reactions at the cobalt-carbon bond of its $\mathrm{B}_{12}$-cofactor, e.g., the relocation of the carboxyl-CoA residue in the interconversion of methylmalonyl-CoA and succinyl-CoA. Such a cobalamin-dependent step might play a role in acetone activation by $D$. biacutus if the (activated?) acetone is carbonylated at the central carbon atom to form first a branched-chain intermediate which subsequently has to be isomerized (linearized).

A very prominent, apparently acetone-inducible protein, spot AS_12 (Figure 1A), identified ORF 04514 in gene cluster $\mathrm{B}$ (Figure $3 \mathrm{~B}$ ); the identification and induction of this gene during growth with acetone was also confirmed by the total proteome analysis. ORF 04514 is predicted to encode a zinc-containing, $\mathrm{NAD}^{+}$-dependent alcohol dehydrogenase (COG1063). A putative gene in direct proximity, ORF 04510 , was attributed to prominent spot AS_19, and is annotated to encode a 'putative redox-active protein' (PF09719) of unknown function; the identification and induction of this gene during growth with acetone was also confirmed by the total proteome analysis. Gene cluster B contains also two genes for benzoyl-CoA reductase subunits, and genes for enoyl-CoA hydratase, 3-hydroxyacyl-CoA dehydrogenase, and acetylCoA acetyltransferase (Figure 3B). The gene for acetylCoA acetyltransferase (04509) was co-identified by spot AS_12, however, at much lower score (Table 2). The gene for enoyl-CoA hydratase (04513) was identified with high score for protein spot AS_18 that appeared on both gels, hence, likely represents a constitutively expressed protein 
Table 2 Identification of proteins observed specifically in extracts of acetone-grown cells of D. biacutus (see Figures 1 and 2 )

\begin{tabular}{|c|c|c|c|}
\hline Spot Id. & IMG locus tag (DebiaDRAFT_) & Annotation & Score \\
\hline AS_3 & 04566 & \$Thiamine diphosphate-requiring enzyme & 652 \\
\hline AM_2 & & & 282 \\
\hline AS_4 & 03619 & ${ }^{\S}$ Adenosine phosphosulfate reductase alpha subunit & 1595 \\
\hline \multirow[t]{2}{*}{ AS_12 } & 04514 & ${ }^{\S} Z n$-dependent dehydrogenases (threonine dehydrogenase) & 970 \\
\hline & 04509 & ${ }^{\S}$ Acetyl-CoA acetyltransferase & 200 \\
\hline AS_17 & 04571 & ${ }^{\S}$ Dehydrogenases with different specificities (related to short-chain alcohol dehydrogenases) & 1944 \\
\hline AS_19 & 04510 & 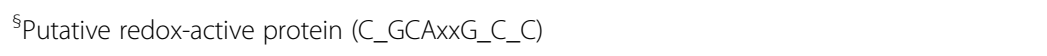 & 1035 \\
\hline AS_25 & 04573 & ${ }^{\S}$ Methylmalonyl-CoA mutase C-terminal domain & 1315 \\
\hline AS_32 & 01796 & Desulfoferredoxin & 449 \\
\hline AM_0 & 04339 & ${ }^{\text {SPyruvate:ferredoxin (flavodoxin) oxidoreductase }}$ & 335 \\
\hline AM_10 & 03042 & 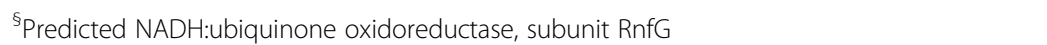 & 159 \\
\hline
\end{tabular}

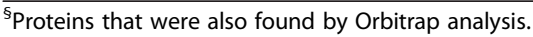

during growth with both acetone and butyrate. Notably, all these genes of gene cluster B (Figure 3B) were identified in the total proteome analysis, except for gene 04512, and gene cluster B appears not to be conserved in any other bacterial genome available within the IMG database thus far.

Another prominent, apparently acetone-inducible protein, AS_32 (Figure 1A), corresponded to ORF 01796 which is predicted to encode a desulfoferredoxin (COG2023). This gene is on a contig with predicted genes (01797-01805) that might code for the following proteins: rubrerythrin, hypothetical protein conserved in SRB, cytochrome $b d$-type quinol oxidase subunit 1 and subunit 2 , multimeric flavodoxin subunit, rubredoxin, uncharacterized flavoprotein (COG0426), ferredoxin, and DsrE/DsrF-like family protein. However, we could identify only a predicted desulfoferredoxin (01796) as highly expressed protein in our gel-based proteomic approach (Table 2). This complex is similar to the one found in Desulfovibrio alaskensis G20 which also contains desulfoferredoxin [23]. The function of the entire complex is not clear, however, it may be involved in the protection of the cells against oxidative stress, e. g., by removing superoxide radicals (see UniProt Q30WF9).

The very prominent, apparently acetone-inducible protein AS_4 (Figure 1A) was encoded by gene 03619 which is predicted to encode the alpha-subunit of adenosine phosphosulfate reductase (AprA). However, this enzyme is relevant for both growth conditions since it is involved in the reduction of adenosine phosphosulfate to sulfite and AMP. This gene is located in a cluster with adenosine phosphosulfate reductase beta-subunit gene (03620), and also this gene was identified, both in acetone- and butyrate-grown cells, for the very prominent spot AS_23 (and AS_24).

A weak, high-molecular weight band AM_0 appeared in the membrane fraction of acetone-grown cells (Figure 2) and corresponded to gene 04339; it is predicted to encode a CoA-acetylating pyruvate:ferredoxin (flavodoxin) oxidoreductase. Finally, band AM_10 which also appeared to be more prominent in acetone-grown cells than in butyrate-grown cells, corresponded to gene 03042 and is predicted to encode a NADH:ubiquinone oxidoreductase subunit RnfG. This gene is located in a predicted operon with the following genes: NADH: ubiquinone oxidoreductase subunit RnfA (03040); electron transport complex Rnf ABCDGE type, B subunit (03039); electron transport complex Rnf ABCDGE type, C subunit (03044); electron transport complex Rnf ABCDGE type, D subunit (03043); NADH: ubiquinone oxidoreductase RnfE subunit (03041). The total proteome analysis confirmed that genes 04339 and 03042 , and, in addition, all other subunit genes of the RnfABCDGE type complex were expressed during growth with acetone and during growth with butyrate (see Additional file 2: Table S1). This Rnf complex might play a role in the supply with low-potential electrons for $\mathrm{CO}_{2}$ reduction to provide $\mathrm{CO}$ as a co-substrate in the initial carbonylation reaction.

\section{Proteins identified for growth with both acetone and butyrate}

The prominent spot AS_4 (see above; Table 2) corresponded to gene 03619 for the alpha-subunit of adenosine phosphosulfate reductase (AprA). This gene is located in a cluster with adenosine phosphosulfate reductase beta-subunit gene 03620, and also this gene was identified for the very prominent spots AS_23 and AS_24 (Figure 1AB, Table 3). The gene cluster contains also the gene (03617) for the subunit $\mathrm{A}$ of heterodisulfide reductase and related polyferredoxins (HdrA). A similar gene cluster was found in the genomes of Desulfovibrio vulgaris DP4, Desulfovibrio vulgaris Hildenborough, and Desulfococcus oleovorans Hxd3. Spot AS_8 (Figure 1AB) 
Table 3 Identification of proteins common in extracts of acetone- and butyrate-grown cells of D. biacutus (see Figures 1 and 2)

\begin{tabular}{|c|c|c|c|}
\hline Band Id. & IMG locus tag (DebiaDRAFT_) & Annotation & Score \\
\hline \multirow[t]{3}{*}{ AS_5 } & 02387 & ${ }^{\text {s} F o r m y l t e t r a h y d r o f o l a t e ~ s y n t h e t a s e ~}$ & 1027 \\
\hline & 03619 & ${ }^{\S}$ Adenosine phosphosulfate reductase alpha subunit & 343 \\
\hline & 03447 & ${ }^{\S} \mathrm{NAD}(\mathrm{P}) \mathrm{H}$-nitrite reductase & 314 \\
\hline AS_6 & 03586 & ${ }^{\S}$ ATP sulphurylase & 1338 \\
\hline AS_7 & & & 1454 \\
\hline AS_8 & 04385 & ${ }^{\S}$ Sulfite reductase alpha subunit (dissimilatory type) & 814 \\
\hline AS_9 & 00156 & ${ }^{\S} \mathrm{ABC}$-type amino acid transport/signal transduction systems periplasmic component/domain & 1367 \\
\hline AS_10 & 02798 & ABC-type amino acid transport/signal transduction systems periplasmic component/domain & 1844 \\
\hline \multirow[t]{2}{*}{ AS_11 } & 01292 & ABC-type amino acid transport/signal transduction systems periplasmic component/domain & 1514 \\
\hline & 03292 & ${ }^{\S}$ Acyl-CoA dehydrogenase & 326 \\
\hline AS_13 & 04384 & §Sulfite reductase beta subunit (dissimilatory type) & 682 \\
\hline AS_14 & 01640 & ${ }^{\S}$ Pterin binding enzyme & 771 \\
\hline AS_15 & 02722 & ${ }^{\S} \mathrm{ABC}$-type amino acid transport periplasmic component & 1168 \\
\hline \multirow[t]{2}{*}{ AS_16 } & 00168 & ${ }^{\S} \mathrm{NAD}$-dependent malate dehydrogenase & 1411 \\
\hline & 02722 & ${ }^{\S} \mathrm{ABC}$-type amino acid transport/signal transduction systems periplasmic component/domain & 245 \\
\hline \multirow[t]{4}{*}{ AS_18 } & 04513 & ${ }^{\text {SEnoyl-CoA hydratase/carnithine racemase }}$ & 847 \\
\hline & 04490 & ABC-type amino acid transport/signal transduction systems periplasmic component/domain & 730 \\
\hline & 01784 & ${ }^{\text {SEnoyl-CoA hydratase/carnitine racemase }}$ & 467 \\
\hline & 03805 & ${ }^{\S}$ Short-chain dehydrogenases of various substrate specificities & 417 \\
\hline AS_23 & 03620 & ${ }^{\S}$ Adenosine phosphosulfate reductase beta subunit & 535 \\
\hline AS_24 & & & 340 \\
\hline AS_36 & 03129 & Uncharacterized conserved protein & 635 \\
\hline AM_1 & 01638 & ${ }^{\S} \mathrm{CO}$ dehydrogenase/CO-methylating acetyl-CoA synthase complex beta subunit & 588 \\
\hline AM_3 & 00010 & ${ }^{\S}$ Acetyl-CoA carboxylase, carboxyltransferase component (subunits alpha and beta) & 397 \\
\hline AM_4 & 03347 & ${ }^{\text {SProton translocating ATP synthase, F1 alpha subunit }}$ & 811 \\
\hline AM_5 & 03345 & ${ }^{\S}$ ATP synthase, F1 beta subunit & 1146 \\
\hline AM_6 & 00583 & Hypothetical protein & 700 \\
\hline AM_7 & 03345 & ${ }^{\S}$ ATP synthase, F1 beta subunit & 204 \\
\hline AM_8 & 03346 & ${ }^{\S}$ ATPsynthase, F1 gamma subunit & 651 \\
\hline AM_9 & 01843 & ${ }_{5,10 \text {-methylenetetrahydrofolate reductase }}$ & 155 \\
\hline AM_11 & 03348 & ${ }^{\S}$ ATP synthase, F1 delta subunit & 607 \\
\hline AM_12 & 03344 & ${ }^{\S}$ ATP synthase, F1 epsilon subunit & 447 \\
\hline
\end{tabular}

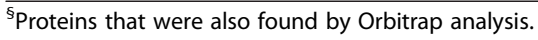

corresponds to gene 04385 which most likely encodes a dissimilatory-type sulfite reductase alpha-subunit (Table 3). The gene is co-located in a cluster with the gene for dissimilatory-type sulfite reductase beta-subunit (04384), which was also identified by PMF (spot AS_13). In the same cluster, genes are located that code for dissimilatory sulfite reductase D (04383) and for NADH: flavin oxidoreductases of the Old Yellow Enzyme family (04379). This cluster is also found in Desulfococcus oleovorans Hxd3, and Desulfovibrio vulgaris Hildenborough, and DP4 strains, and in Desulfovibrio alaskensis G20.
Also all subunit genes of the ATP synthase complex arranged in a gene cluster in the genome of $D$. biacutus were identified in both acetone- and butyrate-grown cells. These identifications were confirmed by the total proteome analysis.

Band AM_1 (Figure 2) was encoded by ORF 01638 which is annotated as $\mathrm{CO}$ dehydrogenase/CO-methylating acetyl-CoA synthase beta subunit gene (Table 3). This subunit gene is encoded in a gene cluster together with candidate genes for $\mathrm{CO}$ dehydrogenase/acetyl-CoA synthase delta subunit (01636), catalytic subunit (01637) 

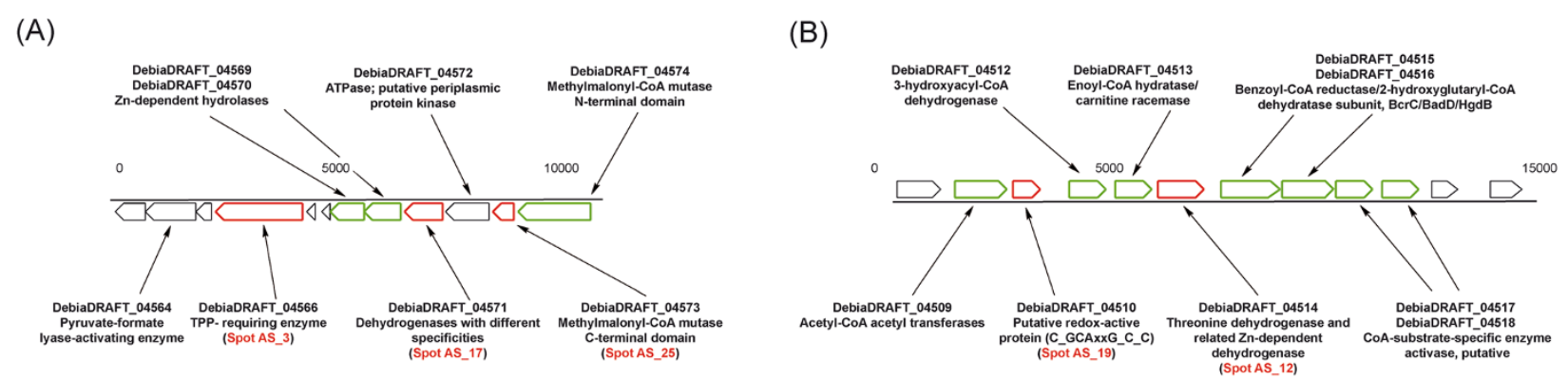

Figure 3 Schematic representation of the two gene clusters (A and B) in D. biacutus harboring genes that appeared to be specifically induced during growth with acetone. Their locus tags in the IMG genome annotation, the corresponding spot numbers on the 2D-PAGE gels, and a description of their annotation are indicated. The acetone-inducible genes identified via the differential 2D-gel-based analysis are labeled in red, and further genes that were identified in a differential total proteome analysis are labeled in green.

and gamma subunit (01639), and candidate genes for a pterin binding enzyme (01640), phosphoenolpyruvate synthase/pyruvate phosphate dikinase (01642), and dehydrogenase (01643). The delta and gamma subunit genes of the $\mathrm{CO}$ dehydrogenase complex were identified in the total proteome analysis (see Additional file 1: Table S1). This enzyme complex is used in D. biacutus for the oxidation of acetyl residues in the Wood-Ljungdahl pathway.

Band AM_3 (Figure 2) was encoded by ORF 00010 which is annotated as an acetyl-CoA/propionyl-CoA/3methylcrotonyl-CoA carboxylase/carboxyltransferase beta component gene (Table 3); this observation was verified in the total proteome analysis (score for acetone, 1173; score for butyrate, 382). The total proteome analysis identified further genes on the same contig (not shown), for acetyl-CoA acetyltransferase (00008), isopropylmalate/homocitrate/citramalate synthase (00007), acyl-CoA synthetase (AMP-forming)/AMP-acid ligases II (00009), acyl-CoA synthetase (NDP forming) (00014), and methylmalonyl-CoA epimerase (00015), each with a higher score for acetone-grown cells than for butyrategrown cells (see Additional file 1: Table S1); interestingly, the co-located gene for a sodium-transporting methylmalonyl-CoA decarboxylase (00011) could not be identified. Whether these enzymes are also involved in acetone metabolism or operate solely in amino acid synthesis remains to be elucidated in the future.

\section{Conclusions}

The results of our proteomic analyses clearly document that $D$. biacutus uses a mechanism for acetone activation that is basically different from the carboxylation reactions observed in aerobic or nitrate-reducing bacteria. The genome does not contain genes coding for enzymes that would be comparable to the described acetone carboxylases [1-3]. Rather, our results indicate that acetone is carbonylated in an ATP and TDP-dependent reaction to an aldehyde derivative which is subsequently oxidized to an acetoacetyl-CoA derivative and further to $\mathrm{CO}_{2}$. The mechanism of the carbonylation reaction remains unclear at present, including the question whether acetoacetaldehyde is the primary product of this reaction. The acetone-specific synthesis of a cobalamine-containing enzyme indicates that a branched-chain derivative may be formed first which is later isomerized to acetoacetaldehyde. In any case, the novel reaction pathway is energetically more favorable for the energy-deprived metabolism of a sulfate-reducing bacterium: It probably requires one ATP equivalent for acetone activation and a further fraction of an ATP equivalent for $\mathrm{CO}_{2}$ reduction to $\mathrm{CO}$ employing an Rnf complex to form acetoacetylCoA. The described pathways for acetone degradation by aerobic or nitrate-reducing bacteria require at least 3 ATP equivalents to obtain this intermediate. One may speculate whether the novel concept of substrate carbonylation is applied also in the activation of other comparably stable compounds.

\section{Methods}

\section{Bacteria and growth conditions}

Desulfococcus biacutus strain KMRActS (DSM 5651) from our own culture collection was grown in freshwater mineral salts medium in $1 \mathrm{~L}$ flasks under an anoxic gas phase $\mathrm{N}_{2} / \mathrm{CO}_{2}(80 / 20)$ at $30^{\circ} \mathrm{C}$ in the dark, as described previously $[7,24]$. The medium was reduced with sodium sulfide, buffered with bicarbonate and adjusted to $\mathrm{pH}$ 7.2, and supplemented with $5 \mathrm{mM}$ acetone or $5 \mathrm{mM}$ butyrate as sole organic carbon source, plus $10 \mathrm{mM}$ sulfate as the electron acceptor.

\section{DNA extraction, sequencing and annotation}

Total DNA was extracted according to the CTAB Protocol for Bacterial DNA extraction of the DoE-JGI (http:// my.jgi.doe.gov) including RNAse treatment. Library preparation, sequencing, and assembly were conducted under 
contract at GATC Biotech AG (Konstanz, Germany). Briefly, two different libraries were prepared, a $3 \mathrm{~kb}$ pairedend library and a shotgun library with an insert size of approximately $1800 \mathrm{bp}$. Both libraries were sequenced using the Roche GS FLX + instrument. The paired-end library was sequenced on 2x 1/16 GS FLX Pico-Titer Plate with GS FLX Titanium XLR70 chemistry, and the shotgun library on 1/2 GS FLX Pico-Titer Plate with GS FLX Titanium $\mathrm{XL}+$ chemistry. Analysis of the readout and de novo assemblies were done with the GS FLX System Software GS De Novo Assembler (Newbler) version 2.6, using the default parameters and the "read flowgrams" (SFF files) of the shotgun library and the $3 \mathrm{~kb}$ paired end library as input. The assembly (153 contigs) was submitted to the IMG annotation pipeline, and the obtained annotation was used to set up a local PMF database at the Proteomics Center of the University of Konstanz.

\section{Preparation of cell-free extracts}

$D$. biacutus cells were collected in the late exponential growth phase $\left(\mathrm{OD}_{600 \mathrm{~nm}} \approx 0.3\right)$ by centrifugation $(6,000 \times g$, $20 \mathrm{~min}, 4^{\circ} \mathrm{C}$ ) and washed three times in Tris- $\mathrm{HCl}$ buffer (30 mM, pH 7.2). The cell pellet was resuspended in Tris$\mathrm{HCl}$ buffer (30 mM, pH 7.2) supplemented with $0.5 \mathrm{mg}$ DNase $\mathrm{mL}^{-1}$ and $1 \mathrm{mg} \mathrm{mL}^{-1}$ of Protease Inhibitor Cocktail (Complete Mini tablets, Roche Diagnostics $\mathrm{GmbH}$, Mannheim, Germany), and cells were disrupted by three passages through a chilled French pressure cell (100 MPa; Aminco). Cell debris was removed by centrifugation $\left(27,000 \times g, 20 \mathrm{~min}, 4^{\circ} \mathrm{C}\right)$ and the membrane fragments collected by ultracentrifugation $(50,000 \times g, 60 \mathrm{~min}$, $\left.4{ }^{\circ} \mathrm{C}\right)$; the supernatant was called the soluble protein fraction. Membrane fragments were washed four times with the same buffer and solubilized with a specific phosphate buffer (see below).

\section{Protein gel electrophoresis}

2D-PAGE of soluble proteins was carried out essentially according to our previously published protocol [25] using the BioRad Ready Strip IPG/Protean II system. Briefly, the soluble protein fraction was desalted by gel filtration (Sephadex $^{\text {тм }}$ G-25 columns, GE Healthcare) and each sample of $1 \mathrm{mg}$ total protein was precipitated by addition of 5 volumes of ice-cold acetone (overnight, $-20^{\circ} \mathrm{C}$ ). The protein precipitate was collected by centrifugation $(13,000 \times g$, $10 \mathrm{~min}, 4^{\circ} \mathrm{C}$ ), the protein pellet air-dried (30 $\left.\mathrm{min}, \mathrm{RT}\right)$, the proteins solubilised in rehydration buffer $(300 \mu \mathrm{L})$ and loaded onto an isoelectric focusing (IEF) strip (BioRad IPG strips, $17 \mathrm{~cm}, \mathrm{pH} \mathrm{5-8).} \mathrm{The} \mathrm{isoelectric} \mathrm{focusing}$ program involved a voltage ramp (rapid) to a maximal voltage of $10,000 \mathrm{~V}$ during $3 \mathrm{~h}$, and a total focusing of 40,000 Volt-hours (Vh). Strips were equilibrated in SDSequilibration buffers I and II (with DTT and iodoacetamide, respectively) and placed onto an SDS-PAGE gel [26] using an overlay of SDS-gel buffer solidified with agarose (0.5\%). The gels $(17 \times 20 \mathrm{~cm}$; BioRad Protean II XI cell $)$ contained a gradient of $5-18 \%$ polyacrylamide in the resolving gel and $4 \%$ polyacrylamide in the stacking gel. Gels were stained with colloidal Coomassie blue R-250 [27]. Protein spots of interest were excised from the gels and submitted to peptide-fingerprinting-mass spectrometry.

1D-PAGE of membrane proteins involved solubilization of the washed membrane fragments (see above) in $200 \mathrm{mM} \mathrm{NaH}{ }_{2} \mathrm{PO}_{4}$ buffer, pH 6.0, supplemented with $150 \mathrm{mM} \mathrm{NaCl}$ and $10 \%$ SDS (w/v), incubation of the protein samples with 2 volumes SDS-gel loading buffer with $5 \% \beta$-mercaptoethanol at $100^{\circ} \mathrm{C}$ for $5 \mathrm{~min}$, prior to loading the samples onto a SDS-PAGE gel; the gels $(17 \times 20 \mathrm{~cm})$ contained a gradient of $5-18 \%$ polyacrylamide in the resolving gel and $4 \%$ polyacrylamide in the stacking gel, and were stained with colloidal Coomassie blue R-250 (see above). Protein bands of interest were excised from the gels and submitted to peptide-fingerprintingmass spectrometry.

For a total proteome analysis of crude extracts (soluble and membrane proteins) from acetone and butyrate grown cells, samples with each $30 \mu \mathrm{g}$ of total protein were mixed with $5 \% \quad \beta$-mercaptoethanol, boiled for $5 \mathrm{~min}$, and loaded onto a small SDS-PAGE gel $(8 \times$ $6 \mathrm{~cm}$, BioRad Protean Mini cell), which contained 4\% polyacrylamide in the stacking gel and $10 \%$ polyacrylamide in the resolving gel. The gel was run until the bands had just entered the resolving gel. The gel was stained with Coomassie blue R-250 (see above), and each band (app. $3 \times 3 \mathrm{~mm}$ ) excised and submitted to high-resolution (Orbitrap) peptide fingerprinting-mass spectrometry.

\section{Peptide fingerprinting-mass spectrometry and database searching}

Protein bands or spots excised from the gels were analyzed at the Proteomics Facility of the University of Konstanz (www.proteomics-facility.uni-konstanz.de). All tryptic digests were analyzed by reversed-phase liquid chromatography tandem mass spectrometry (LC-MS/ MS) using an Esquire 3000 mass spectrometer (Bruker Daltonics), connected to an Agilent 1100 HPLC. After sample injection, the column was washed for $5 \mathrm{~min}$ with $90 \%$ mobile phase A ( $0.1 \%$ formic acid) and $10 \%$ mobile phase B ( $0.1 \%$ formic acid in acetonitrile), and peptides were eluted using a linear gradient of $10 \%$ mobile phase $\mathrm{B}$ to $80 \%$ mobile phase $\mathrm{B}$ in $20 \mathrm{~min}$ at $50 \mu \mathrm{l} \mathrm{min}{ }^{-1}$. The Esquire mass spectrometer was operated in a datadependent mode in which each full MS scan was followed by three MS/MS scans where the three most abundant molecular ions were dynamically selected and fragmented by collision-induced dissociation (CID). Dynamic exclusion was allowed. 
The MASCOT engine (Matrix Science, London, UK) was used to match each peptide fingerprint against a local database of all predicted protein sequences of the annotated genome of Desulfococcus biacutus KMRAcS (IMG, see above), and against the external EMBL and NCBI databases. The parameters for searching and scoring were as follows. One missed cleavage site allowed. Fixed modifications: carbamidomethyl Cys. Variable modifications: N-term. pyro-Glu, N-term. Gln, Met-oxidation. Peptide charge: $2^{+}, 3^{+}, 4^{+}$. Peptide tolerance: $1.0 \mathrm{Da}$. MS/MS tolerance: $0.8 \mathrm{Da}$. If not stated otherwise (see Results), a minimal score of 200 and/or minimal sequence coverage of $30 \%$ was set as cut-off for low-scoring hits.

\section{Availability of supporting data}

Data supporting the proteomics results are included as Additional file 1: Table S1 and Additional file 2: Table S2) within the article. The draft genome annotation used in this study is available within the IMG platform (https:// img.jgi.doe.gov) under IMG Submission ID 7648.

\section{Additional files}

Additional file 1: Table S1. Results of the total proteome analysis of crude extract of $D$. biacutus grown with acetone.

Additional file 2: Table S2. Results of the total proteome analysis of crude extract of $D$. biacutus grown with butyrate.

\section{Abbreviations}

PMF: Peptide mass fingerprinting; 2D-PAGE: Two-dimensional polyacrylamide gel electrophoresis; ORF: Open reading frame.

\section{Competing interests}

The authors declare that they have no competing interests.

\section{Authors' contribution}

OGA, DS and BS conceived and designed the study. OGA and DS carried out the genomic and proteomic analyses. OGA, DS and BS carried out the analysis of the data, and wrote the manuscript. All authors read and approved the final manuscript.

\section{Acknowledgements \\ We thank GATC Biotech AG (sequencing), Andreas Marquardt (Proteomics Facility), and our colleagues at University of Konstanz for their support. We are grateful for funds from the Deutsche Forschungsgemeinschaft (DFG) in its SPP 1319 priority program. Work by OGA was also supported by the Konstanz Research School Chemical Biology (KoRS-CB), and work by DS by a DFG grant (SCHL1936/1), the research funds of the University of Konstanz, and by the Konstanz Young Scholar Fund (YSF).}

Received: 21 March 2014 Accepted: 3 July 2014

Published: 11 July 2014

\section{References}

1. Sluis MK, Small FJ, Allen JR, Ensign SA: Involvement of an ATP-dependent carboxylase in a CO2-dependent pathway of acetone metabolism by Xanthobacter strain Py2. J Bacteriol 1996, 178(14):4020-4026.

2. Schühle $K$, Heider J: Acetone and butanone metabolism of the denitrifying bacterium "Aromatoleum aromaticum" demonstrates novel biochemical properties of an ATP-dependent aliphatic ketone carboxylase. J Bacteriol 2012, 194(1):131-141.
3. Dullius $\mathrm{CH}$, Chen $\mathrm{CY}$, Schink B: Nitrate-dependent degradation of acetone by Alicycliphilus and Paracoccus strains and comparison of acetone carboxylase enzymes. Appl Environ Microbiol 2011, 77(19):6821-6825.

4. Sluis MK, Larsen RA, Krum JG, Anderson R, Metcalf WW, Ensign SA: Biochemical, molecular, and genetic analyses of the acetone carboxylases from Xanthobacter autotrophicus strain Py2 and Rhodobacter capsulatus strain B10. J Bacteriol 2002, 184(11):2969-2977.

5. Janssen PH, Schink B: Metabolic pathways and energetics of the acetoneoxidizing, sulfate-reducing bacterium, Desulfobacterium cetonicum. Arch Microbiol 1995, 163(3):188-194.

6. Platen H, Schink B, Temmes A: Anaerobic degradation of acetone by Desulfococcus biacutus spec. nov. Arch Microbiol 1990, 154(4):355-361.

7. Gutiérrez Acosta OB, Hardt N, Schink B: Carbonylation as a key reaction in anaerobic acetone activation by Desulfococcus biacutus. Appl Environ Microbiol 2013, 79(20):6228-6235.

8. DiDonato RJ Jr, Young ND, Butler JE, Chin KJ, Hixson KK, Mouser P, Lipton MS, DeBoy R, Methe BA: Genome sequence of the deltaproteobacterial strain NaphS2 and analysis of differential gene expression during anaerobic growth on naphthalene. PLoS One 2010, 5(11):e14072.

9. Wohlbrand L, Jacob JH, Kube M, Mussmann M, Jarling R, Beck A, Amann R, Wilkes H, Reinhardt R, Rabus R: Complete genome, catabolic sub-proteomes and key-metabolites of Desulfobacula toluolica Tol2, a marine, aromatic compound-degrading, sulfate-reducing bacterium. Environ Microbiol 2013, 15(5):1334-1355.

10. Strittmatter AW, Liesegang H, Rabus R, Decker I, Amann J, Andres S, Henne A, Fricke WF, Martinez-Arias R, Bartels D, Goesmann A, Krause L, Puhler A, Klenk HP, Richter M, Schuler M, Glockner FO, Meyerdierks A, Gottschalk G, Amann R: Genome sequence of Desulfobacterium autotrophicum HRM2, a marine sulfate reducer oxidizing organic carbon completely to carbon dioxide. Environ Microbiol 2009, 11(5):1038-1055.

11. Callaghan AV, Morris BE, Pereira IA, Mclnerney MJ, Austin RN, Groves JT, Kukor JJ, Suflita JM, Young LY, Zylstra GJ, Wawrik B: The genome sequence of Desulfatibacillum alkenivorans AK-01: a blueprint for anaerobic alkane oxidation. Environ Microbiol 2012, 14(1):101-113.

12. Rabus R, Ruepp A, Frickey T, Rattei T, Fartmann B, Stark M, Bauer M, Zibat A, Lombardot T, Becker I, Amann J, Gellner K, Teeling H, Leuschner WD, Glockner FO, Lupas AN, Amann R, Klenk HP: The genome of Desulfotalea psychrophila, a sulfate-reducing bacterium from permanently cold Arctic sediments. Environ Microbiol 2004, 6(9):887-902.

13. Spring S, Lapidus A, Schroder M, Gleim D, Sims D, Meincke L, Glavina De Rio T, Tice H, Copeland A, Cheng JF, Lucas S, Chen F, Nolan M, Bruce D, Goodwin L, Pitluck S, Ivanova N, Mavromatis K, Mikhailova N, Pati A, Chen A, Palaniappan K, Land M, Hauser L, Chang YJ, Jeffries CD, Chain P, Saunders $E$, Brettin T, Detter JC, et al: Complete genome sequence of Desulfotomaculum acetoxidans type strain (5575). Stand Genomic SCi 2009, 1(3):242-253.

14. Sun H, Spring S, Lapidus A, Davenport K, Del Rio TG, Tice H, Nolan M, Copeland A, Cheng JF, Lucas S, Tapia R, Goodwin L, Pitluck S, Ivanova N, Pagani I, Mavromatis K, Ovchinnikova G, Pati A, Chen A, Palaniappan K, Hauser L, Chang YJ, Jeffries CD, Detter JC, Han C, Rohde M, Brambilla E, Goker M, Woyke T, Bristow J, et al: Complete genome sequence of Desulfarculus baarsii type strain (2st14). Stand Genomic Sci 2010, 3(3):276-284

15. Aeckersberg F, Bak F, Widdel F: Anaerobic oxidation of saturated hydrocarbons to $\mathrm{CO} 2$ by a new type of sulfate-reducing bacterium. Arch Microbiol 1991, 156:5-14.

16. Kondo $\mathrm{H}$, Ishimoto $\mathrm{M}$ : Requirement for thiamine pyrophosphate and magnesium for sulfoacetaldehyde sulfo-lyase activity. J Biochem 1974, 76(1):229-231.

17. Pierce $E$, Becker DF, Ragsdale SW: Identification and characterization of oxalate oxidoreductase, a novel thiamine pyrophosphate-dependent 2-oxoacid oxidoreductase that enables anaerobic growth on oxalate. J Biol Chem 2010, 285(52):40515-40524.

18. Reed L: Multienzyme complexes. Acc Chem Res 1974, 7:40-46.

19. Kochetov GA, Izotova AE: Interactions of thiamine pyrophosphate and transketolase. Dokl Akad Nauk SSSR 1972, 205:986-988.

20. Lindqvist $Y$, Schneider G, Ermler U, Sundstrom M: Three-dimensional structure of transketolase, a thiamine diphosphate dependent enzyme, at 2.5 A resolution. EMBO J 1992, 11(7):2373-2379.

21. Kluger R: Thiamin diphosphate: a mechanistic update on enzymic and nonenzymic catalysis of decarboxylation. Chem $\operatorname{Rev} 1987,87(5): 863-876$. 
22. Gutiérrez Acosta OB, Hardt N, Hacker SM, Strittmatter T, Schink B, Marx A: Thiamine pyrophosphate stimulates acetone activation by Desulfococcus biacutus as monitored by a fluorogenic ATP analogue. ACS Chem Biol 2014, 9(6):1263-1266.

23. Hauser L, Land ML, Brown SD, Larimer F, Keller KL, Rapp-Giles BJ, Price MN, Lin M, Bruce DC, Detter JC, Tapia R, Han CS, Goodwin LA, Cheng JF, Pitluck S, Copeland A, Lucas S, Nolan M, Lapidus AL, Palumbo AV, Wall JD: Complete genome sequence and updated annotation of Desulfovibrio alaskensis G20. J Bacteriol 2011, 193(16):4268-4269.

24. Janssen PH, Schink B: Catabolic and anabolic enzyme activities and energetics of acetone metabolism of the sulfate-reducing bacterium Desulfococcus biacutus. J Bacteriol 1995, 177(2):277-282.

25. Schmidt A, Müller N, Schink B, Schleheck D: A proteomic view at the biochemistry of syntrophic butyrate oxidation in Syntrophomonas wolfei. PLoS One 2013, 8(2):e56905.

26. Laemmli UK: Cleavage of structural proteins during assembly of head of bacteriophage-T4. Nature 1970, 227(5259):680-685.

27. Neuhoff $V$, Arold N, Taube D, Ehrhardt W: Improved staining of proteins in polyacrylamide gels including isoelectric focusing gels with clear background at nanogram sensitivity using Coomassie Brilliant Blue G-250 and R-250. Electrophoresis 1988, 9(6):255-262.

doi:10.1186/1471-2164-15-584

Cite this article as: Gutiérrez Acosta et al: Acetone utilization by sulfatereducing bacteria: draft genome sequence of Desulfococcus biacutus and a proteomic survey of acetone-inducible proteins. BMC Genomics 2014 15:584.

\section{Submit your next manuscript to BioMed Central and take full advantage of:}

- Convenient online submission

- Thorough peer review

- No space constraints or color figure charges

- Immediate publication on acceptance

- Inclusion in PubMed, CAS, Scopus and Google Scholar

- Research which is freely available for redistribution 